\title{
MadFlow: towards the automation of Monte Carlo simula- tion on GPU for particle physics processes
}

\author{
Stefano Carrazza ${ }^{1,2,3, *}$, Juan Cruz-Martinez ${ }^{1, * *}$, Marco Rossi ${ }^{1,2, * * *}$, and Marco Zaro ${ }^{1, * * * *}$ \\ ${ }^{1}$ Dipartimento di Fisica, Università degli Studi di Milano and INFN Sezione di Milano, Milan, Italy \\ ${ }^{2}$ CERN, Theoretical Physics Department and OpenLab, CH-1211 Geneva 23, Switzerland. \\ ${ }^{3}$ Quantum Research Centre, Technology Innovation Institute, Abu Dhabi, UAE.
}

\begin{abstract}
In this proceedings we present MadFlow, a new framework for the automation of Monte Carlo (MC) simulation on graphics processing units (GPU) for particle physics processes. In order to automate MC simulation for a generic number of processes, we design a program which provides to the user the possibility to simulate custom processes through the MadGraph5_aMC@NLO framework. The pipeline includes a first stage where the analytic expressions for matrix elements and phase space are generated and exported in a GPU-like format. The simulation is then performed using the VegasFlow and PDFFlow libraries which deploy automatically the full simulation on systems with different hardware acceleration capabilities, such as multithreading CPU, single-GPU and multi-GPU setups. We show some preliminary results for leading-order simulations on different hardware configurations.
\end{abstract}

\section{Introduction and motivation}

In the last years we have observed a quickly growing interest on the development of new code frameworks based on hardware accelerators, in particular graphics processing units (GPU), for the improvement of performance and efficiency in scientific and industrial problems.

There are several examples in High Energy Physics (HEP) applications that can benefit from a systematic implementation (or conversion) of existing codes and algorithms on GPU. Some examples have already been successfully published, such as deep learning applications in experimental physics [1], where spectacular performance improvements were obtained thanks to the employment of GPUs.

The HEP community has been very active in the field during the last years, for example providing computational tools for experimental setups. On the other hand, we still observe a growing trend towards the increase of computational time required to solve complex problems [2,3] in particular for Monte Carlo (MC) simulation of particle physics processes and observables. In fact, public MC simulation libraries rely, almost exclusively, on CPU technology [4-7].

In order to write a full parton-level MC competitive by any measure with existing tools at least four ingredients need to be produced: an integrator library able to parallelize over the

\footnotetext{
*e-mail: stefano.carrazza@unimi.it

**e-mail: juan.cruz@mi.infn.it

***e-mail: marco.rossi@cern.ch

****e-mail: marco.zaro@mi.infn.it
} 
number of events; a GPU-capable PDF interpolation tool; an efficient phase space generator, which should not only generate valid phase space points on GPU but also apply any fiducial cuts and finally the matrix element squared for the target processes are required.

In the last year, we have developed tools that provide the ground basis for the implementation of an automatic Monte Carlo simulation tool for HEP addressing some of the aforementioned issues: VegasFlow [8,9] and PDFFlow [10, 11]. VegasFlow is a new software for fast evaluation of high dimensional integrals based on Monte Carlo integration techniques designed for platforms with hardware accelerators. It enables developers to delegate all complicated aspects of hardware or platform implementation to the library, so they can focus on the problem at hand. PDFFlow is a library which provides fast evaluation of parton distribution functions (PDFs) designed for platforms with hardware accelerators.

In this proceedings we address the issue of the generation of GPU-ready matrix elements. We present MadFlow, a framework which provides an automatic pipeline for GPU deployment of Monte Carlo simulation for custom processes, by combining the matrix elements and phase space expressions generated by the MadGraph5_aMC@NLO [5, 6] framework with the VegasFlow and PDFFlow efficient simulation tool for hardware accelerators. MadFlow will be deployed as a new open-source library, opening the possibility to study and benchmark multiple approaches to Monte Carlo integration based on distributed hardware, and, in future, new algorithms based on deep learning techniques.

This draft is structured as follows. In Section 2 we describe the technical implementation of our framework. In Section 3 we compare and benchmark results. Finally, in Section 4 we present our conclusion and future development roadmap.

\section{Technical implementation}

\subsection{The MadFlow design}

We appreciate that many groups nowadays rely on very extensive code bases and learning to use an equally complicated framework (no matter the benefit) might not be feasible for everyone. Therefore, in order to accelerate the adoption of hardware accelerators within the HEP-ph community our main concerns when designing a Monte Carlo implementation on GPU have been the maintainability of the framework and making it as developer-friendly as possible.

In order to achieve our goals, we consider the MadGraph5_aMC@NLO framework (MG5_aMC) [5, 6] as the entry point our procedure. MG5_aMC is a meta-code written in Python, that generates automatically the code in a low-level language to be employed for the simulation of arbitrary scattering processes at colliders, in the Standard Model or beyond. MG5_aMC relies on general procedures and methods, without being tailored to a specific process, class of processes, or physics model. Besides the generation of tree-level matrix elements, MG5_aMC gives also the possibility to the user to include Next-to-Leading order corrections, both due to strong and electroweak interactions (including matching to partonshower for the former). However, in this paper we will limit ourselves to the case of tree-level matrix elements.

The workflow of MG5_aMC is the following: a model, written in the Universal Feynrules Output (UFO) format [12], is loaded, which contains all the informations on the underlying theory, including the Feynman rules. Starting from the model, Feynman diagrams are generated, and the corresponding expressions for the matrix elements are written in processspecific files. The various parts of the Feynman diagrams (external wavefunctions, vertices, propagators, etc.) are evaluated via the ALOHA routines [13] (with the introduction of MadGraph5 [14] ALOHA supersedes the HELAS routines [15]). 


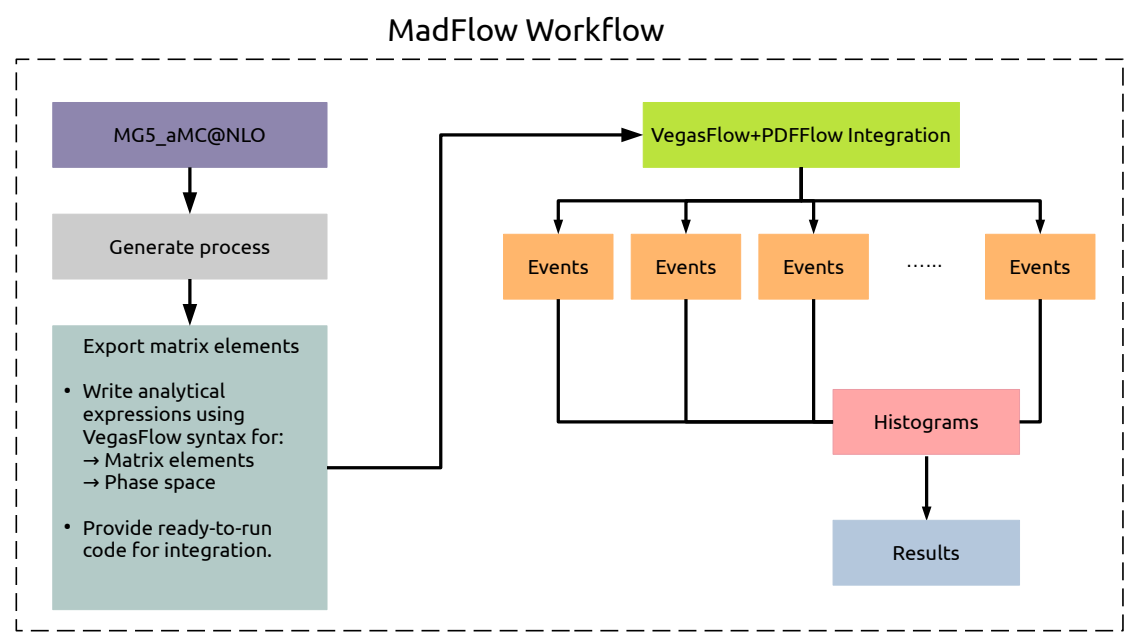

Figure 1. Schematic workflow for the implementation of MadFlow. The process starts directly from MG5_aMC, where the user generates a custom process using the standard framework API and exports the relevant code in a specific format for VegasFlow and PDFFlow integration.

It is then natural to consider a GPU extension of MG5_aMC (and of of ALOHA) as the basis of our future general purpose parton-level GPU MC generator. For consistency with the current string of names, we have dubbed this framework with the provisional MadFlow. We should note there is an independent effort dedicated to porting MG5_aMC to GPU [16]. The MadFlow project differs from "Madgraph 4 GPU" for two main reasons: the interest in providing the VegasFlow integration engine and thus the possibility to deploy the algorithm on multiple hardware accelerators, and the need of a technical solution which simplifies maintainability and does not require specialized GPU knowledge from the developer and user point of view.

In Figure 1 we show schematically the stages involved in the implementation of MadFlow. The process starts directly from MG5_aMC, where the user generates a custom process using the standard framework API and exports the relevant code for the analytic matrix elements and phase space expressions in python, using the syntax defined by VegasFlow. In terms of code implementation this step requires the development of a MG5_aMC plugin, which consists of an exporter module to write the matrix element and the ALOHA routines in Python, fulfilling the requirements imposed by VegasFlow and PDFFlow using TensorFlow [17] primitives. The main difficulty consists in converting sequential functions into vectorized functions. During the MadFlow development we have performed several numerical and performance benchmarks in order to avoid potential bugs.

After the conversion step into the specified format is performed, the exported python code is incorporated into a VegasFlow device-agnostic integration algorithm which executes the event generation pipeline from the generation of random numbers, computation of the phase space kinematics, matrix element evaluation and histogram accumulation.

\subsection{The evaluation of matrix elements routines}

The evaluation of the matrix elements in MadFlow follows the original MG5_aMC implementation: a Matrix class is produced by the Python exporter plugin module. Its smatrix 


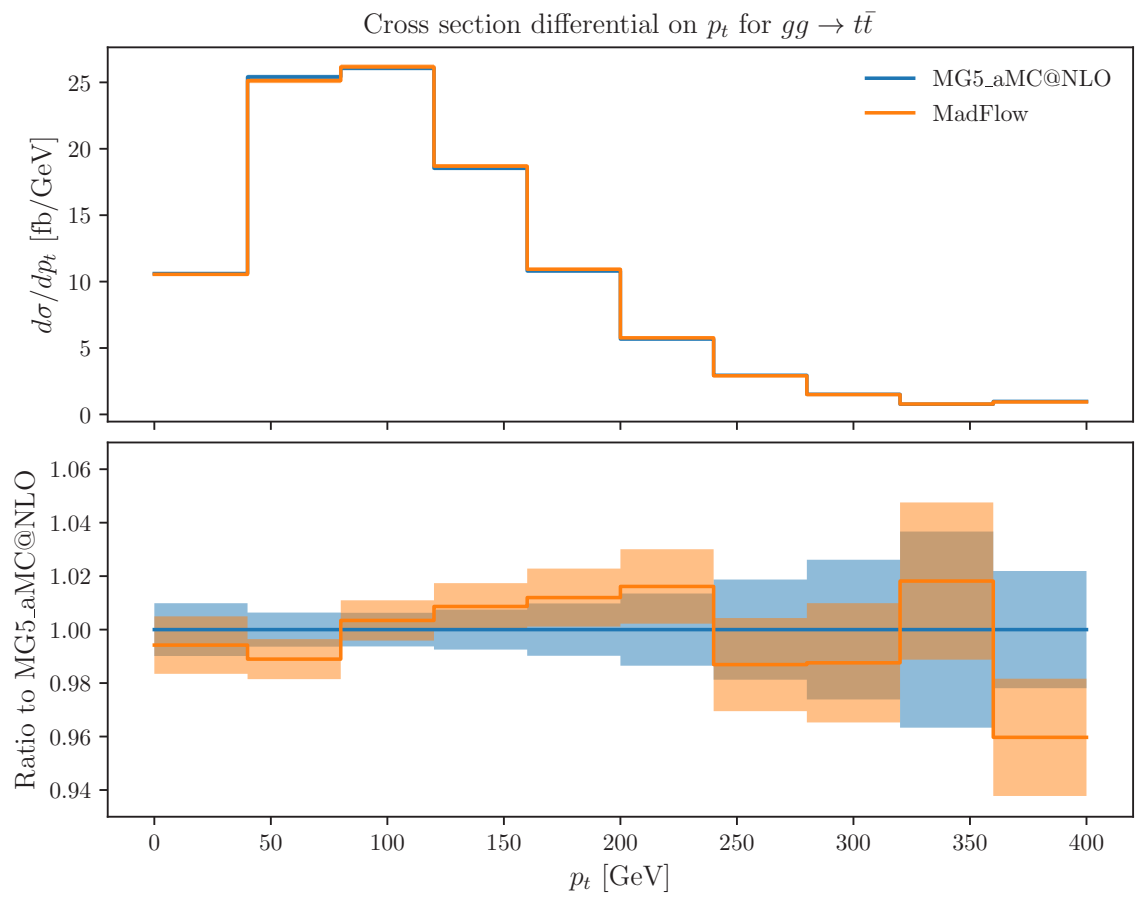

Figure 2. Leading Order cross section differential on $p_{t}$ for gluon-gluon fusion at $\sqrt{s}=14 \mathrm{TeV}$. We compare predictions between MG5_aMC (blue) and VegasFlow (orange), using the conversion tool described in Section 2. The two cross sections are in statistical agreement.

method links together the needed Feynman rules to compute the requested matrix element: it loops over initial and final state particle helicities and aggregates their contribution to the final squared amplitude.

The matrix element vectorization requires to replace the ALOHA waveforms and vertices routines abiding by the TensorFlow ControlFlow rules. Although this process is straightforward for vertices Feynman rules, being mostly comprised by elementary algebraic operations, the implementation of particle waveforms functions is subject to several conditional control statements that definitely make the task harder.

\section{Preliminary results}

We have tested the pyout extension to the MG5_aMC exporter for GPU-ready code in a Leading Order calculation for gluon-gluon fusion at $\sqrt{s}=14 \mathrm{TeV}$. Note that, in principle, any process generated by the MG5_aMC framework can be integrated by the MadFlow library.

\subsection{Accuracy}

In Figure 2 we show the Leading Order cross section differential on $p_{t}$ for gluon-gluon fusion for predictions obtained with the original MG5_aMC integration procedure and the new pyout approach based on VegasFlow and PDFFlow. The plot in the first row shows the differential distribution in absolute scale in $\mathrm{fb} / \mathrm{GeV}$, while the plot in the second row show 


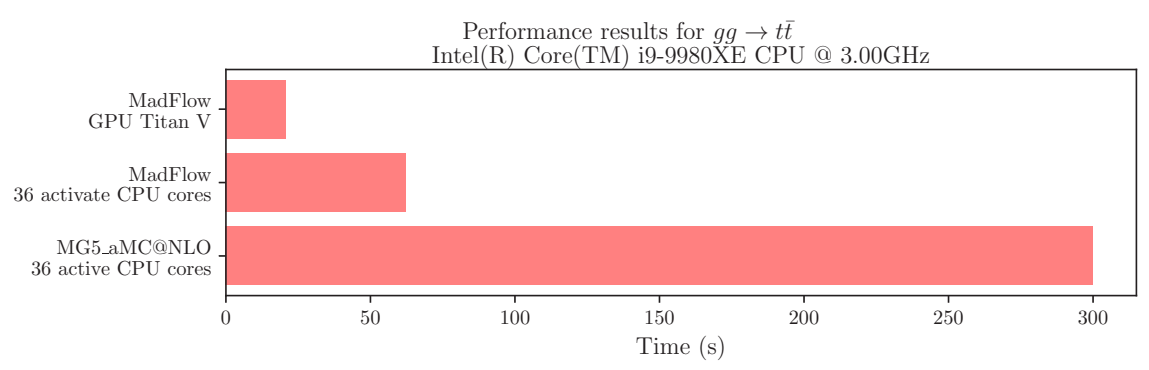

Figure 3. Comparison of a Leading Order cross section differential on $p_{t}$ for gluon-gluon fusion at $\sqrt{s}=14 \mathrm{TeV}$ ran in both VegasFlow and MG5_aMC. The CPU-only version of VegasFlow is able to improve the performance obtained by MG5_aMC for the same level of target accuracy due mainly to a difference on the parallelization implementation of both codes. The usage of the GPU device further improves the performance.

the ratio between both computations, confirming a good level of agreement between both implementations for the same level of target accuracy of $0.02 \%$.

The results presented here are computed independently from each framework in order to minimize communication bottlenecks between CPU-GPU, in fact for this particular configuration MadFlow does not require any specific asynchronous CPU evaluation, thus all computations are performed directly on GPU and results stored on GPU memory.

\subsection{Performance}

In terms of performance, in particular evaluation time, in Figure 3 we compare the total amount of time required by the simulation using three different configurations based on a workstation with an Intel i9-9980XE CPU with 36 logical cores. The first horizontal bar shows the greatest performance of MadFlow running on a single NVIDIA Titan V GPU with $12 \mathrm{~GB}$ of RAM. The second bar shows the timings for the same code evaluated just on the CPU chip using all available cores. The last bar shows the equivalent simulation performance using the MG5_aMC default integrator. We conclude that the MadFlow implementation confirms a great performance improvement when running on GPU hardware.

\section{Outlook}

In conclusion in this proceedings we propose a new approach for the generalization of Monte Carlo simulation on hardware accelerators. In particular, the MadFlow design provides a fast and maintainable code which can quickly port complex analytic expressions into hardware specific languages without complex operations involving several computer languages, tools and compilers. Furthermore, preliminary results confirm the algorithm effectiveness, providing benefits in terms of performance when compared to CPU based simulations.

As an outlook, we plan to release a development version of MadFlow as an open-source library, and proceed with the implementation of all required changes to accomodate Next-toLeading order computations.

\section{Acknowledgements}

S.C. and J.C.M are supported by the European Research Council under the European Union's Horizon 2020 research and innovation Programme, grant agreement number 740006, valid 
until the end of 2021, exclusively for research topics related to the NNNPDF project. M.Z. is supported by Programma per Giovani Ricercatori "Rita Levi Montalcini" granted by the Italian Ministero dell’Università e della Ricerca (MUR).

\section{References}

[1] K. Albertsson et al., J. Phys. Conf. Ser. 1085, 022008 (2018), 1807.02876

[2] S. Hoche et al., Working Group Report: Computing for Perturbative QCD, in Community Summer Study 2013: Snowmass on the Mississippi (2013), 1309. 3598

[3] J. Niehues, D.M. Walker, Phys. Lett. B 788, 243 (2019), 1807.02529

[4] T. Gleisberg, S. Hoeche, F. Krauss, M. Schonherr, S. Schumann, F. Siegert, J. Winter, JHEP 02, 007 (2009), 0811 . 4622

[5] J. Alwall, R. Frederix, S. Frixione, V. Hirschi, F. Maltoni, O. Mattelaer, H.S. Shao, T. Stelzer, P. Torrielli, M. Zaro, JHEP 07, 079 (2014), 1405.0301

[6] R. Frederix, S. Frixione, V. Hirschi, D. Pagani, H.S. Shao, M. Zaro, JHEP 07, 185 (2018), 1804.10017

[7] J. Campbell, T. Neumann, JHEP 12, 034 (2019), 1909.09117

[8] S. Carrazza, J.M. Cruz-Martinez, Comput. Phys. Commun. 254, 107376 (2020), 2002.12921

[9] S. Carrazza, J.M. Cruz-Martinez, VegasFlow: accelerating Monte Carlo simulation across platforms, in 40th International Conference on High Energy Physics (2020), 2010.09341

[10] S. Carrazza, J.M. Cruz-Martinez, M. Rossi (2020), 2009.06635

[11] M. Rossi, S. Carrazza, J.M. Cruz-Martinez, PDFFlow: hardware accelerating parton density access, in 40th International Conference on High Energy Physics (2020), 2012.08221

[12] C. Degrande, C. Duhr, B. Fuks, D. Grellscheid, O. Mattelaer, T. Reiter, Comput. Phys. Commun. 183, 1201 (2012), 1108.2040

[13] P. de Aquino, W. Link, F. Maltoni, O. Mattelaer, T. Stelzer, Comput. Phys. Commun. 183, 2254 (2012), 1108. 2041

[14] J. Alwall, M. Herquet, F. Maltoni, O. Mattelaer, T. Stelzer, JHEP 06, 128 (2011), 1106.0522

[15] H. Murayama, I. Watanabe, K. Hagiwara (1992)

[16] Madgraph 4 gpu, https://github.com/madgraph5/madgraph4gpu

[17] M. Abadi, A. Agarwal, P. Barham, E. Brevdo, Z. Chen, C. Citro, G.S. Corrado, A. Davis, J. Dean, M. Devin et al., TensorFlow: Large-scale machine learning on heterogeneous systems (2015), software available from tensorflow.org, http:// tensorflow.org/ 